\title{
Uma Análise da Influência da Flexibilização do Trabalho sobre a Satisfação do Trabalhador e o Comportamento de Cidadania Organizacional
}

\author{
An Analysis of the Influence of Flexible Work Arrangements on Employee Satisfaction and \\ Organizational Citizenship Behavior \\ Harrison Bachion Ceribeli ${ }^{1}$, Thaisa Prado Mignacca*1 \\ ${ }^{1}$ Universidade Federal de Ouro Preto (IFOP), Ouro Preto, Brasil.
}

\begin{tabular}{l} 
I N F O A R T I G O \\
\hline Palavras-chave: \\
Horário de Trabalho Flexível, \\
Home-Office, \\
Cidadão Organizacional, \\
Satisfação no Trabalho.
\end{tabular}

A R T ICLE INFO

\section{Keywords:}

Flexible Working Hours,

Home-Office,

Organizational Citizen,

Job Satisfaction.

\begin{abstract}
R E S U M O
À medida que as organizações passaram a adequar as características do trabalho para atender as expectativas e anseios dos funcionários, surgiu a necessidade de compreender como isso afeta diferentes dimensões do comportamento humano no trabalho. Neste contexto, objetivou-se, com a presente pesquisa, analisar como a flexibilização do trabalho influencia a satisfação do trabalhador e como ambas afetam o comportamento de cidadania organizacional. O método de pesquisa utilizado foi a survey e, para análise dos dados, foi empregada a técnica de Modelagem de Equações Estruturais. Analisando os resultados obtidos, constatou-se que o comportamento de cidadania organizacional é influenciado positivamente pela satisfação do trabalhador e negativamente pela flexibilização do trabalho. Ademais, verificou-se que os arranjos laborais flexíveis impactam positivamente a satisfação no trabalho. Com isso, contribui-se para o avanço da compreensão acerca dos antecedentes da cidadania organizacional e, ao mesmo tempo, dos impactos da flexibilização do trabalho.

A B S T R A C T
Organizations began to adapt the characteristics of the work to meet the expectations
and desires of its employees. Consequently, it became necessary to understand how
this affects different dimensions of human behavior at work. In this context, this study
aimed to analyze how the flexibilization of work influences worker satisfaction and
how both affect organizational citizenship behavior. The research method used was the
survey and, for analysis of the data, the Structural Equation Modeling technique was
used. Analyzing the obtained results, it was verified that organizational citizenship
behavior is influenced positively by the satisfaction of the worker and negatively by
the flexibility of the work. In addition, it was found that flexible working arrangements
positively impact job satisfaction. With these results, this research contributes to the
advancement of the understanding about the antecedents of organizational citizenship
and, at the same time, the impacts of the flexibilization of work.
\end{abstract}

\section{Introdução}

O termo CCO (comportamento de cidadania organizacional) foi cunhado por Smith, Organ e Near (1983) e Bateman e Organ (1983) a partir da observação de condutas de cooperação voluntária nas organizações. Esses mesmos autores definiram tal construto como um comportamento dos trabalhadores que engloba a realização, de forma espontânea, de atividades extras, que extrapolam as responsabilidades dos cargos ocupados, suprindo necessidades organizacionais e, ao mesmo tempo, auxiliando colegas de trabalho.

A partir de então, passou a ser atribuída cada vez maior importância a esse construto, o que se deve aos múltiplos benefícios que a ele foram associados, os quais favorecem tanto os indivíduos diretamente afetados pela postura dos colegas cidadãos organizacionais quanto as próprias organizações (WILLIAMS; ANDERSON, 1991).

\footnotetext{
* Principal contato para correspondência.

Ceribeli, H. B. (harrisonbceribeli@hotmail.com), Mignacca, T. P. (thaisamignacca@gmail.com).
}

DOI: $\underline{\text { dx.doi.org/10.21714/1679-18272019v17Ed.p88-104 }}$

1679-1827 C 2019 Gest@o.org. 
Dado o impacto positivo que o CCO exerce direta ou indiretamente sobre as organizações, um número crescente de pesquisas dedicou-se a analisar seus antecedentes, tais como o suporte e apoio dos líderes (SMITH; ORGAN; NEAR, 1983), a satisfação do trabalhador e sua percepção de justiça organizacional (PODSAKOFF et al., 2000), a cultura organizacional (ARDA et al., 2017), o suporte organizacional percebido pelos funcionários (READER et al., 2017), o cumprimento do contrato psicológico (NGUYEN et al., 2016), a resiliência (PAUL; BAMEL; GARG, 2016) e a flexibilização do trabalho (BAEZA; WANG; WANG, 2017).

Entretanto, por se tratar de um construto multifacetado, fazem-se necessários mais estudos abordando tais antecedentes, muitos dos quais ainda não foram devidamente explorados na literatura (PAUL; BAMEL; GARG, 2016). Em vista disso, objetivou-se, com esta pesquisa, analisar como a flexibilização do trabalho influencia a satisfação do trabalhador e como ambas afetam o comportamento de cidadania organizacional.

A justificativa para a realização da presente investigação reside, primeiramente, na escassez de trabalhos, tanto em âmbito nacional quanto internacional, que se propuseram a examinar como os arranjos laborais flexíveis afetam a conduta dos trabalhadores no ambiente laboral.

Ademais, destaca-se também a importância de se analisar como a adoção de práticas como o home-office e a flexibilização dos horários impacta a satisfação do trabalhador, que, além de ser um fator desencadeador de melhora no clima organizacional (VIITALA; TANSKANEN; SÄNTTI, 2015), ainda afeta diversas dimensões do comportamento humano nas organizações.

\section{Referencial Teórico}

\subsection{Flexibilização do Trabalho}

A dificuldade que as organizações passaram a enfrentar para atrair e reter talentos tem estimulado os gestores a pensarem em diferentes alternativas para suprir as demandas dos funcionários e adequar as características do trabalho às expectativas e anseios deles (WONG; WAN; GAO, 2017).

Neste sentido, pode-se apontar como um dos principais desafios dos gestores na atualidade a adoção de políticas e mecanismos que levem ao aumento da satisfação dos funcionários, assim como à substituição das tradicionais condições laborais, marcadas pela rigidez e padronização, por um sistema de trabalho capaz de atender às reivindicações individuais dos trabalhadores (DE LA TORRE-RUIZ; VIDAL-SALAZAR; CORDÓN-POZO, 2017).

Para isso, faz-se necessária a implantação de práticas que flexibilizem o trabalho, o que, segundo Bajzikova et al. (2013), tornar-se-á uma condição imprescindível para que as organizações permaneçam competitivas no mercado, contando com os melhores profissionais.

A flexibilização do trabalho, que envolve a flexibilização do horário e/ou redução da jornada laboral dos indivíduos, assim como a adoção do home-office e do teletrabalho (POSSENRIEDE; HASSINK; PLANTENGA, 2016), permite que os funcionários escolham o melhor lugar e momento para realizarem suas tarefas e ainda harmonizem sua vida pessoal e a profissional (WHEATLEY, 2017).

Tal prática tende a ser benéfica para as organizações, pois, além de garantir maior competitividade por favorecer a atração e retenção de talentos, ainda possibilita a contratação de indivíduos que não têm disponibilidade ou mesmo interesse por um trabalho em jornada integral, como mulheres com crianças, estudantes e aposentados (BAJZIKOVA et al., 2013).

No caso específico das mulheres com crianças, Noback, Broersma e Dijk (2016) chamam a atenção para o fato de que a flexibilização do trabalho, especialmente a redução da jornada, contribui para a conciliação da vida profissional com a pessoal, favorecendo a carreira dessas profissionais que também são mães, que necessitam de tempo para educar e cuidar dos filhos e, por isso, muitas vezes dão preferência a um emprego de meio período.

Entre as práticas de flexibilização do trabalho, além da redução da jornada, tem-se a flexibilização dos horários, também denominada de flex-time, que se baseia na concessão de maior autonomia ao funcionário para definir o começo e o término de sua jornada laboral diária, desde que seja cumprida a carga horária total previamente acordada, trazendo como vantagens a ampliação da produtividade dos trabalhadores, a consolidação do comprometimento com a organização, o desenvolvimento individual e a retenção dos bons profissionais (MARTIN; MACDONNELL, 2012).

Outra alternativa para flexibilizar o trabalho envolve a possibilidade de o indivíduo exercer suas atividades laborais longe do ambiente organizacional, sem o contato físico com os demais profissionais, o que pode se dar por meio do home-office, quando as tarefas são realizadas em domicílio, ou do teletrabalho, que demanda, 
necessariamente, meios tecnológicos para execução não presencial das tarefas (RAFALSKI; ANDRADE, 2015).

De qualquer forma, independente do modelo escolhido, é importante que seja obtida uma relação ganhaganha, favorável tanto para os indivíduos quanto para as organizações, ou seja, que possibilite ao sujeito balancear sua vida pessoal e o trabalho e ter tempo para suas atividades pessoais, familiares e profissionais, o que diminui conflitos e estresse, ao mesmo tempo em que traga benefícios para as organizações, o que inclui o aumento da produtividade individual, a atração e retenção dos melhores profissionais, a redução do uso dos planos de saúde, a queda no absenteísmo e a diminuição das horas extras pagas (WHEATLEY, 2012).

É importante acrescentar que essas práticas, que têm resultado em mudanças nas tradicionais demarcações de local e tempo de trabalho, são consequência das transformações que vêm ocorrendo nas tecnologias e no ambiente corporativo, que viabilizam maior controle por parte dos gestores e, ao mesmo tempo, autonomia para os trabalhadores (COLE; OLIVER; BLAVIESCIUNAITE, 2014).

Ainda assim, notam-se algumas desvantagens associadas à flexibilização no trabalho, envolvendo tanto as jornadas de meio período quanto o home-office e o teletrabalho, como a falta de visibilidade e interação presencial do funcionário, além da menor socialização com superiores e pares (RAFALSKI; ANDRADE, 2015), que podem refletir na perda de oportunidades de networking e de capacitação, e até mesmo dificultar a promoção na carreira (MCDONALD; BRADLEY; BROWN, 2009).

Mas, apesar dessas desvantagens, os resultados alcançados tendem a ser vantajosos para as partes envolvidas, afetando de maneira positiva o comportamento dos indivíduos nas organizações, melhorando o desempenho deles no trabalho e favorecendo a retenção de talentos (COENEN; KOK, 2014). Para isso, todavia, a flexibilização deve ser implantada de forma cuidadosa e bem planejada, levando em consideração a natureza do trabalho, a função exercida pelo profissional, o perfil e o nível de qualificação do sujeito (MOCELIN, 2011). Caso contrário, pode impactar de forma negativa no funcionamento da organização e rendimento do funcionário (WHEATLEY, 2017).

\subsection{Satisfação do Trabalhador}

Há diferentes definições para o conceito de satisfação no trabalho. Lee, Ok e Hwang (2016) o definem como algo subjetivo e individual, que resulta da confrontação entre as expectativas do trabalhador e as reais condições e características do trabalho. Na mesma linha, Spector (2006) define a satisfação do trabalhador como um posicionamento inconstante que depende de como ele percebe, em dado momento, aspectos de seu trabalho.

A satisfação no trabalho também é concebida como um estado afetivo e/ou atitude cognitiva derivada da avaliação que o sujeito faz acerca de suas experiências laborais (BRIEF, 1998), ou ainda como uma sensação de prazer ou estado emocional positivo ligado à percepção individual a respeito do trabalho (LEE; OK; HWANG, 2016).

Existem diversos fatores que influenciam diretamente o nível de satisfação dos trabalhadores, tais como a remuneração (MONTERO; VASQUEZ, 2015), o ambiente de trabalho (VIITALA; TANSKANEN; SÄNTTI, 2015), a carreira (BOATENG; OKOE; MENSAH, 2017), a autonomia (COENEN; KOK, 2014) e a justiça percebida (GEVREK et al., 2017).

Mas, além dos aspectos supracitados, é importante considerar também a influência positiva que a flexibilização laboral exerce sobre a satisfação do trabalhador, à medida que propicia a este último conciliar as diferentes dimensões de sua vida (HUMPHREY; NAHRGANG; MORGESON, 2007; COLE; OLIVER; BLAVIESCIUNAITE， 2014; POSSENRIEDE; HASSINK; PLANTENGA， 2016; POSSENRIEDE; PLANTENGA, 2014; STAVROU, 2005; WHEATLEY, 2017). Neste sentido, tem-se a primeira hipótese da pesquisa:

- H1: a flexibilização dos arranjos laborais impacta positivamente a satisfação do trabalhador.

Com a flexibilização dos arranjos laborais tornando-se cada vez mais recorrente nas organizações, os funcionários deixam de estar vinculados ao ambiente de trabalho tradicional e adquirem liberdade para definir quando e onde exercer suas funções (COENEN; KOK, 2014). Com isso, eles são capazes de gerir melhor o tempo e ajustar suas responsabilidades profissionais com atividades pessoais e demandas familiares (LU; CHANG, 2014).

Logo, as necessidades experimentadas dentro e fora do trabalho são supridas, proporcionando uma satisfação holística, que, além de impactar positivamente a produtividade organizacional (WHITMAN; VAN ROOY; VISWESVARAN, 2010), também aumenta o bem-estar e a saúde mental dos indivíduos (OLANIYAN; HYSTAD, 2016). Ou seja, a satisfação no trabalho afeta a saúde dos trabalhadores e influencia seus comportamentos e atitudes, o que justifica a crescente atenção que o tema vem recebendo (MIAO; HUMPHREY; QIAN, 2017). 
Conclui-se, deste modo, que garantir a satisfação dos trabalhadores tem um efeito ganha-ganha: os funcionários experimentam menor estresse, maior bem-estar e mais entusiasmo no trabalho (VIITALA; TANSKANEN; SÄNTTI, 2015); por sua vez, a organização obtém benefícios como a redução dos índices de absenteísmo e rotatividade (TUNA et al. 2016) e a melhoria do clima organizacional (VIITALA; TANSKANEN; SÄNTTI, 2015), além de passar a contar com profissionais mais produtivos e empenhados, que tendem a assumir tarefas e funções que extrapolam as obrigações específicas do cargo que ocupam (BOATENG; OKOE; MENSAH, 2017), ou seja, que se comportam como genuínos cidadãos organizacionais (BELWALKAR; VOHRA; PANDEY, 2018; HERMININGSIH; KASURI, 2018; PIO; TAMPI, 2018; GYEKYE; HAYBATOLLAHI, 2015; JUNG; YOON, 2015; KAUR; KAUR; DHAR, 2015). Sendo assim, delimitou-se como segunda hipótese de pesquisa que:

- H2: a satisfação do trabalhador afeta positivamente o comportamento de cidadania organizacional.

\subsection{Comportamento de Cidadania Organizacional}

Segundo Rose (2016), devido às constantes mudanças e consequente dinamização do local de trabalho, as pesquisas nas áreas administrativa, comportamental e psicológica têm ampliado o foco sobre o comportamento de cidadania organizacional (CCO).

Na literatura, esse construto tende a ser associado a diferentes concepções. Na visão de Arda et al. (2017), sustentada por Smith, Organ e Near (1983), o CCO remete às ações e atividades extras desempenhadas voluntariamente pelo trabalhador, as quais ultrapassam as obrigações definidas formalmente pelo cargo ocupado por ele. Nesta mesma linha, Mayfield e Mayfield (2014) apontam que esse tipo de comportamento é notado quando o colaborador identifica e internaliza os valores da organização naturalmente, exercendo atividades sobressalentes à sua função sem foco na remuneração.

Por outro lado, partindo de uma perspectiva distinta, Marinova, Moon e Dyne (2010) argumentam que o CCO não é considerado como parte extra, mas como uma conduta pertencente à função desempenhada pelo profissional.

O comportamento de cidadania organizacional está ligado a diferentes atitudes e condutas manifestadas pelos funcionários no ambiente de trabalho, as quais compõem dimensões distintas, como altruísmo, virtude cívica e desportivismo (ARDA et al., 2017), ou ainda cortesia (ZHANG, 2014) e consciência (JENA; GOSWAMI, 2014).

De acordo com Arda et al. (2017), altruísmo é descrito como a conduta que consiste em ajudar outras pessoas sem haver necessariamente um retorno, o que desencadeia cooperação no ambiente laboral e, consequentemente, maior eficácia dos grupos; já o exercício da virtude cívica remete ao indivíduo que, independentemente do cargo que ocupa, preocupa-se em ser ativo em diversas atividades na organização; por sua vez, o desportivismo caracteriza quem está disposto a se sujeitar a situações incômodas, mantendo uma atitude positiva, para favorecer a equipe, ou seja, alguém tomado de grande espírito coletivista.

De forma complementar, a cortesia engloba as ações que visam prevenir problemas futuros aos colegas de trabalho, notificando-os e mantendo-os informados (ZHANG, 2014), enquanto a consciência remete ao funcionário cuja atuação excede as funções mínimas exigidas pelo cargo ocupado (JENA; GOSWAMI, 2014; WANG et al., 2013).

Neste sentido, verifica-se que as dimensões do CCO referem-se, basicamente, a um padrão comportamental de solicitude e suporte aos colegas de trabalho, apresentado de forma espontânea pelo indivíduo e, muitas vezes, sem reciprocidade (ROSE, 2016).

Segundo Michel (2017), os funcionários apresentam maior cidadania organizacional quando experimentam mais ânimo e disposição pessoais, quando esperam criar uma imagem favorável deles mesmos junto a seus colegas de trabalho e quando se preocupam em cuidar da organização e de sua equipe como "bons soldados".

Todavia, o comportamento de cidadania organizacional também pode ser desencadeado por alguns elementos ligados ao ambiente laboral, como a existência de um contrato psicológico bem definido, no qual são supridas as expectativas de ambas as partes, que estimula, por exemplo, atitudes altruístas por parte dos funcionários (NGUYEN et al., 2016). Além disso, quando os indivíduos sentem-se valorizados pela organização, assumem papeis extras de forma altruísta e desportivista (GUPTA; SHAHEEN; REDDY, 2017).

Além dos fatores supramencionados, outros antecedentes do CCO incluem a percepção de suporte organizacional (READER et al., 2017) e a predominância de uma cultura pautada no coletivismo, sendo que ambos influenciam os funcionários a ultrapassarem as responsabilidades pré-definidas pelo cargo ocupado (ARDA et al., 2017).

Por fim, a liderança também é apontada como um dos aspectos que estimulam o CCO, pois, à medida que o líder 
encoraja outros funcionários a se desenvolverem, melhora o relacionamento nas equipes de trabalho (ZHANG; GUO; NEWMAN, 2017).

Em vista de todos esses antecedentes, verifica-se que cabe aos próprios gestores adotarem políticas de gestão que promovam o suporte e a justiça organizacional e, por conseguinte, induzam o comportamento de cidadania organizacional, o que engloba, por exemplo, valorizar e tratar os funcionários com respeito e dignidade, para que eles se sintam em um ambiente laboral justo (LIM; LOOSEMORE, 2017).

Complementarmente, outra forma de induzir o CCO é adotar uma política de flexibilização dos arranjos laborais e concessão de maior autonomia aos indivíduos (BAEZA; WANG; WANG, 2017; MAYFIELD; MAYFIELD, 2014), pois, ao ter a possibilidade de escolher como, onde e quando trabalhar, o profissional tende a desenvolver uma boa relação com a organização e a nutrir uma imagem positiva dela, mostrando-se mais disposto a ser um cidadão organizacional (YADAV; RANGNEKAR; BAMEL, 2016). Logo, tem-se a terceira hipótese que norteou a condução desta pesquisa:

- H3: a flexibilização dos arranjos laborais impacta positivamente o comportamento de cidadania organizacional.

Importante mencionar que, de acordo com Rose (2016), a flexibilização laboral, ao mesmo tempo em que apresenta uma relação positiva com o CCO, torna-se um desafio para sua manifestação, à medida que esse comportamento muitas vezes é estimulado pelo convívio e proximidade física dos indivíduos. Nessa mesma linha, Martinez e Gómez (2013) assinalam que, em decorrência da flexibilização dos arranjos laborais, os profissionais que permanecem menos tempo na organização podem acabar "esquecidos", recebendo menos oportunidades de treinamento e desenvolvimento, o que causa um impacto negativo sobre o CCO.

Muitos autores que tratam do tema na atualidade delimitam dois grupos de benefícios resultantes do CCO: um que se refere aos ganhos diretos auferidos pela organização e outro que engloba os ganhos que, primeiramente, afetam os trabalhadores e acabam impactando a organização de forma indireta (MULDOON; KEOUGH; LIGUORI, 2017; ROBERTSON; BARLING, 2017; ROSE, 2016; WEIKAMP; GÖRITZ, 2016).

Entre os benefícios diretos do comportamento de cidadania organizacional, destacam-se o funcionamento mais eficaz das equipes (LIM; LOOSEMORE, 2017), a redução de desperdícios (WANG et al., 2013) e do absenteísmo (MAYFIELD; MAYFIELD, 2014) e a satisfação dos clientes em decorrência da maior produtividade dos funcionários (MICHEL, 2017); em síntese, a melhoria do funcionamento da organização. Já entre os benefícios indiretos do CCO, é possível citar o maior comprometimento desenvolvido pelos indivíduos no local de trabalho, o apoio mútuo vivenciado dentro das equipes e o cooperativismo que leva ao compartilhamento de conhecimento entre os profissionais, elementos que criam um ambiente que favorece a atração e retenção de talentos (ROSE, 2016).

\section{Aspectos Metodológicos}

A presente pesquisa pode ser classificada como descritiva, à medida que teve como objetivo descrever as relações entre os construtos estudados (COOPER; SCHINDLER, 2014), de natureza quantitativa, pois foram coletados dados numéricos, que, posteriormente, receberam tratamento estatístico (HAIR JUNIOR et al., 2005).

O método de coleta de dados empregado foi a survey, que consiste na aquisição de dados primários junto aos indivíduos estudados (HAIR JR et al., 2005), utilizando-se um questionário como instrumento de pesquisa (FREITAS et al., 2000). Segundo Lutkemeyer Filho, Vaccaro e Freitas (2015), esse método é proficiente pela sua simplicidade e pela facilidade de verificação e análise dos dados obtidos; no caso da survey eletrônica, as respostas são arquivadas automaticamente (SANTOS; ANTONELLI, 2011), o acesso às unidades amostrais não depende da proximidade geográfica e ainda abrange mais respondentes em menos tempo (WALTER, 2013).

$\mathrm{O}$ instrumento de pesquisa foi estruturado com base na escala Likert, que permite ao respondente apontar, numericamente, quanto cada afirmativa corresponde à sua realidade (COOPER; SCHINDLER, 2014), tendo como vantagens a facilidade de aplicação, a igualdade psicométrica entre os extremos (MAZARO et al., 2014), além da redução da subjetividade das respostas (FORTE; MATOS; OLIVEIRA, 2015).

Adotou-se uma escala de sete pontos, sendo apenas os extremos nomeados como "concordo totalmente" e "discordo totalmente". Importante esclarecer que a opção por sete pontos teve como intuito aumentar a exatidão da escala de mensuração sem ampliar em demasia a complexidade de preenchimento do ponto de vista dos respondentes, balanceando esses dois aspectos (HAIR JR et al., 2005).

O questionário foi dividido em quatro seções. A primeira foi constituída por questões demográficas para 
posterior caracterização da amostra, enquanto as três seções subsequentes incluíram afirmativas abordando a satisfação no trabalho, a flexibilização dos arranjos laborais e o comportamento de cidadania organizacional.

Para mensurar a satisfação do trabalhador, utilizaram-se quatro questões, retiradas de Jung e Yoon (2017). Para mensurar a flexibilização do trabalho, foram empregadas nove afirmativas já utilizadas por Ceribeli e Ferreira (2016). Por fim, o comportamento de cidadania organizacional foi mensurado por 17 questões, extraídas de Marinova, Moon e Dyne (2010).

A população-alvo do estudo incluiu os trabalhadores habitantes das cidades de Mariana e Ouro Preto, localizadas no Estado de Minas Gerais, que estavam empregados no momento da coleta de dados, independentemente do grau de flexibilização dos arranjos laborais nas empresas onde trabalhavam. Tal delimitação justifica-se considerando o intuito de analisar como uma maior ou menor flexibilização do trabalho afeta a satisfação do profissional e seu comportamento de cidadania organizacional.

Para compor a amostra da pesquisa, optou-se pela técnica de amostragem não probabilística por conveniência, ou seja, a seleção das unidades amostrais foi feita a partir do acesso dos pesquisadores. Essa escolha metodológica fundamentou-se na impossibilidade de listar todos os elementos que fazem parte da população-alvo do estudo, inviabilizando uma escolha aleatória, assim como no baixo custo e na rapidez para coleta dos dados (VIANA, 2011).

Para definir o tamanho da amostra, seguiu-se a recomendação de Hair JR et al. (2009) de que o número de unidades amostrais deve ser de, pelo menos, 10 vezes o número de variáveis da pesquisa. Sendo assim, considerando que foram adotadas 30 variáveis, delimitou-se que a amostra deveria ser composta por 300 respondentes.

A coleta dos dados deu-se de forma presencial, por meio da aplicação de questionários impressos em locais de grande fluxo de trabalhadores nas cidades de Mariana e Ouro Preto, e também eletrônica, utilizando-se um questionário virtual elaborado no Google Forms, cujo link de acesso foi divulgado por meio de redes sociais (Facebook e Whatsapp) e e-mail.

Para análise dos dados, empregou-se a técnica de Modelagem de Equações Estruturais, testando-se um modelo completo de variáveis latentes, que compreende um modelo de mensuração e um modelo estrutural (BYRNE, 2016). O modelo de mensuração retratou o agrupamento das variáveis observadas do estudo em variáveis latentes que representaram os construtos flexibilização do trabalho, satisfação do trabalhador e comportamento de cidadania organizacional. Por sua vez, o modelo estrutural descreveu a ligação entre as variáveis latentes criadas, de acordo com as hipóteses de pesquisa definidas, conforme Figura 1.

Figura 1: Hipóteses da pesquisa.

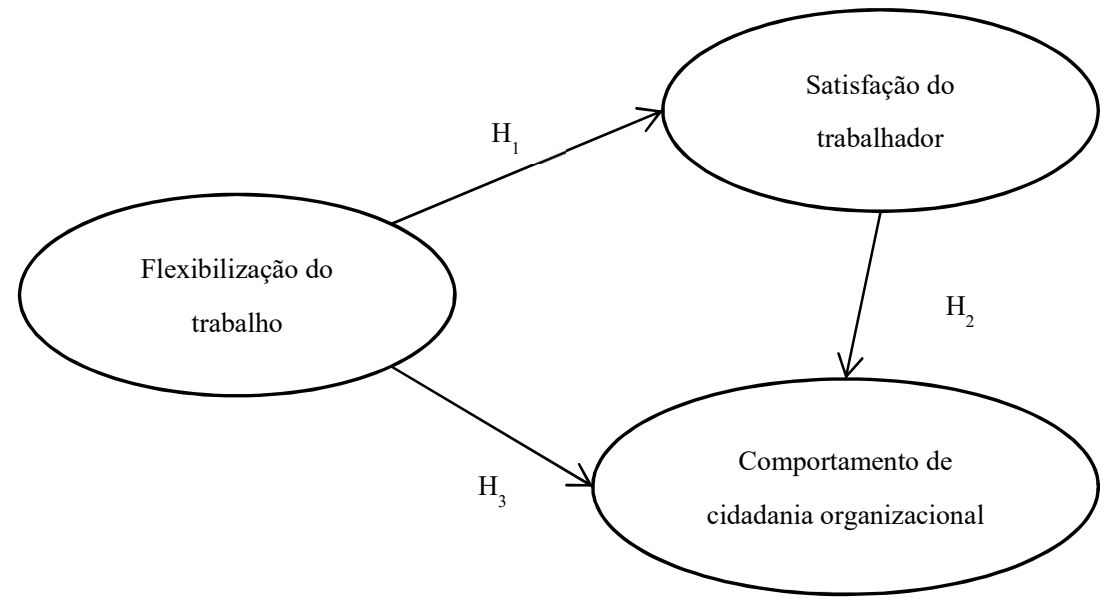

Fonte: Elaboração própria. 


\section{Apresentação e Discussão dos Resultados}

A pesquisa contou com uma amostra composta por 301 respondentes, cujas características são sintetizadas na Tabela 1.

Tabela 1. Caracterização da Amostra.

\begin{tabular}{|c|c|c|}
\hline Variável & Característica & Percentual da amostra \\
\hline \multirow{2}{*}{ Sexo } & Masculino & $39,33 \%$ \\
\hline & Feminino & $60,67 \%$ \\
\hline \multirow{4}{*}{ Faixa etária } & Até 29 anos & $43,67 \%$ \\
\hline & Entre 30 e 39 anos & $30 \%$ \\
\hline & Entre 40 e 49 anos & $12 \%$ \\
\hline & 50 anos ou mais & $14,33 \%$ \\
\hline \multirow{3}{*}{ Estado civil } & Solteiro(a) & $54,67 \%$ \\
\hline & Casado(a) & $35,67 \%$ \\
\hline & Outros & $9,66 \%$ \\
\hline \multirow{3}{*}{ Número de fillhos } & Nenhum & $61,67 \%$ \\
\hline & Um & $20 \%$ \\
\hline & Dois ou mais & $18,33 \%$ \\
\hline \multirow{4}{*}{ Escolaridade } & Ensino superior completo & $40,67 \%$ \\
\hline & Ensino superior incompleto & $17,67 \%$ \\
\hline & Pós-graduação & $34,33 \%$ \\
\hline & Ensino médio & $7,33 \%$ \\
\hline \multirow{3}{*}{$\begin{array}{l}\text { Porte da empresa onde } \\
\text { trabalha }\end{array}$} & Empresa de grande porte & $41,67 \%$ \\
\hline & Empresa de médio porte & $24 \%$ \\
\hline & Micro ou pequena empresa & $34,33 \%$ \\
\hline
\end{tabular}

Fonte: Elaboração própria.

Depois de efetuar a caracterização da amostra, conduziu-se uma análise da normalidade dos dados, com o intuito de verificar se as respostas obtidas para cada variável seguiam o padrão de uma distribuição normal. Para isso, utilizaram-se duas medidas: assimetria, cujo valor deveria estar dentro do intervalo de $-1,96 e+1,96$ (HAIR JR et al., 2009), e curtose, que não deveria extrapolar o intervalo de -3 e+3 (HAIR JR et al., 2005). Utilizando tais parâmetros, decidiu-se pela exclusão das variáveis CCO15 (Faço sugestões inovadoras para melhorar o departamento ou o setor onde trabalho) e SAT4 (Sinto que meu trabalho é significativo).

Em seguida, testou-se, utilizando o software AMOS, um modelo contendo três variáveis latentes: flexibilização do trabalho, comportamento de cidadania organizacional e satisfação do trabalhador, compostas, respectivamente, por nove, 16 e três variáveis observadas. Todavia, houve problemas de identificação do modelo.

Nesse caso, conforme recomendado por Hair JR et al. (2009), seria necessário eliminar alguns dos coeficientes estimados, o que levou à realização de uma Análise Fatorial Exploratória (AFE) englobando, um a um, os conjuntos de variáveis utilizados para mensurar os três construtos estudados.

A partir da AFE que incluiu as noves variáveis relacionadas ao constructo flexibilização do trabalho, observou-se que a variável FLEX9 (Faltas e eventuais atrasos podem ser compensados através de bancos de horas e/ou horas extras) apresentou comunalidade inferior ao parâmetro mínimo aceitável de 0,5 (FIGUEIREDO FILHO; SILVA JR, 2010), no valor de 0,416, o que resultou em sua exclusão.

Posteriormente, na AFE que contemplou as 16 variáveis relacionadas ao construto comportamento de cidadania organizacional, verificou-se que as variáveis CCO2 (Ajudo os colegas de trabalho que estão sobrecarregados) e CCO16 (Atuo como pacificador quando há desentendimentos) deveriam ser agrupadas, cada uma, em um único fator; com isso, ambas foram excluídas. Realizou-se, então, nova AFE com as 14 variáveis restantes, constatando-se que a variável CCO17 (Ajudo a encontrar uma solução quando há desentendimentos 
entre colegas na organização) apresentou comunalidade de 0,394 , ocasionando sua exclusão. Na terceira AFE, alcançou-se uma solução fatorial contendo três fatores, sendo eles "foco em melhoria", "conscienciosidade" e "disposição para ajudar", agrupando, respectivamente, cinco, quatro e quatro variáveis.

Por fim, na AFE que contou com as três variáveis relacionadas à satisfação do trabalhador, foram obtidas apenas comunalidades acima de 0,5 e um único fator, não sendo necessária nenhuma exclusão.

Finalizadas as análises fatoriais exploratórias, testou-se um novo modelo, composto por cinco variáveis latentes, sendo uma exógena (flexibilização do trabalho) e quatro endógenas (satisfação do trabalhador, foco em melhoria, conscienciosidade e disposição para ajudar).

Entretanto, ao se analisar a validade convergente, constatou-se que a variável latente endógena disposição para ajudar apresentou uma variância extraída abaixo do valor de referência de 0,5 (HAIR JR et al., 2009), em decorrência da reduzida carga fatorial da variável CCO1 (Ajudo meus colegas de trabalho quando eles estão ausentes), que alcançou valor de 0,419 . Dessa forma, houve também a exclusão dessa variável.

À vista disso, testou-se um novo modelo estrutural, cuja representação pode ser visualizada na Figura 2.

Figura 2: Modelo de equações estruturais testado.

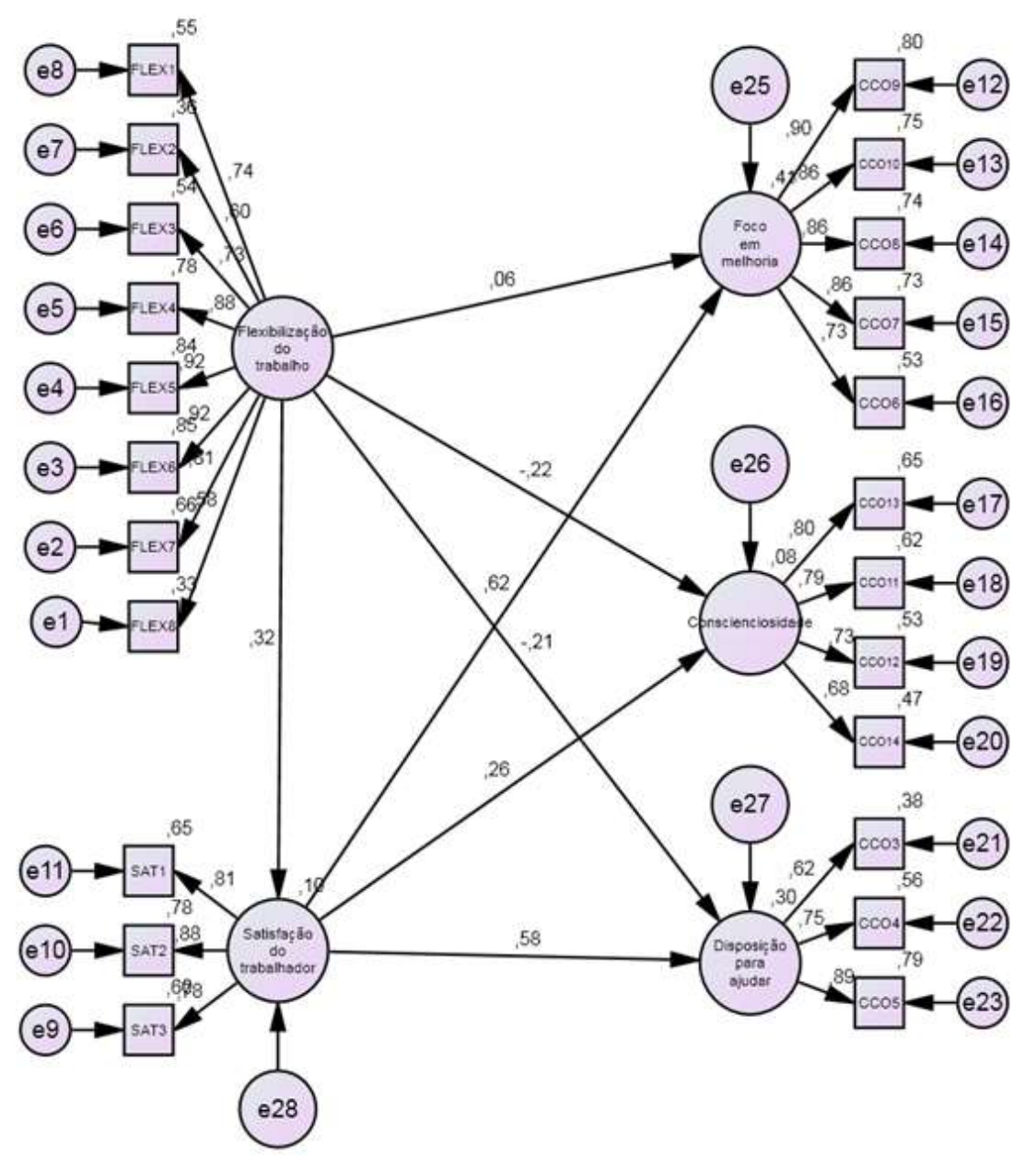

Fonte: Elaboração própria no software AMOS.

Neste ponto, antes de prosseguir com a apresentação dos resultados, é importante esclarecer que, apesar de terem sido excluídas sete variáveis do conjunto original, a análise não foi comprometida, à medida que os construtos investigados mantiveram-se representados por três ou mais itens, evitando distorções relacionadas ao ajuste obtido (HAIR JR et al., 2009).

Preliminarmente à análise das estimativas padronizadas obtidas e da significância estatística das relações propostas, examinou-se a qualidade do modelo, sendo utilizadas, para isso, quatro medidas: a raiz do erro 
quadrático médio de aproximação (RMSEA - Root Mean Square Error of Approximation), o índice de qualidade de ajuste (GFI - Goodness of Fit Index), o índice de qualidade de ajuste calibrado (AGFI - Adjusted Goodness of Fit Index) e o qui-quadrado normado $\left(\chi^{2} / \mathrm{DF}\right)$.

Para o RMSEA, que representa o quão bem o modelo testado se ajusta à população estudada, levando em considerando tanto o tamanho amostral quanto a complexidade do próprio modelo (HAIR JR et al., 2009), são recomendados valores entre 0,05 e 0,08 .

Para o GFI, que mede a quantidade de variância ou covariância que é predita pela matriz reproduzida (SCHUMACKER; LOMAX, 2010), e o AGFI, que é ajustado pelos graus de liberdade do modelo (HAIR JUNIOR et al., 2009), são aceitos, respectivamente, valores próximos ou superiores a 0,9 (SCHUMACKER; LOMAX, 2010) e superiores a 0,8 (STACCIARINI; PACE, 2017).

Finalmente, para o $\chi^{2} / \mathrm{DF}$, que expressa a razão entre o qui-quadrado (medida de ajuste absoluto que permite identificar se existe semelhança entre as matrizes observada e reproduzida) e os graus de liberdade do modelo, são recomendados valores dentro do intervalo entre 1 e 3 (HAIR JR et al., 2009).

Para o modelo testado, foram encontrados os seguintes índices: RMSEA $=0,068$; GFI $=0,85 ;$ AGFI $=0,81$; e $\chi^{2} / \mathrm{DF}=2,377$. Analisando conjuntamente os quatro valores obtidos, ratifica-se que o modelo delineado possui qualidade de ajuste satisfatória, o que justifica a continuação da análise.

Com o intuito de validar especificamente o modelo de mensuração, examinaram-se os $p$-valores das relações entre as variáveis observadas e as respectivas variáveis latentes nas quais elas foram agrupadas. Adotando-se um nível de confiança de $95 \%$, confirmou-se que todos os agrupamentos propostos apresentaram significância estatística, à medida que os $p$-valores obtidos foram inferiores a 0,05 .

Analisaram-se também a variância extraída e a confiabilidade composta relativas a cada variável latente, cujos valores devem ser, respectivamente, superiores a 0,5 e 0,7 (HAIR JR et al., 2009). Na Tabela 2, são apresentados os valores calculados, que permitiram corroborar a validade convergente do modelo.

Tabela 2. Medidas de validade convergente do modelo testado.

\begin{tabular}{ccc} 
Variável Latente & Variância Extraída & Confiabilidade Composta \\
\cline { 2 - 3 } Flexibilização do trabalho & 0,613088 & 0,985994 \\
Satisfação do trabalhador & 0,678172 & 0,983752 \\
Foco em melhoria & 0,711957 & 0,974795 \\
Conscienciosidade & 0,565929 & 0,972216 \\
Disposição para ajudar & 0,575831 & 0,957791 \\
\hline
\end{tabular}

Fonte: Elaboração própria.

Em vista da confiabilidade composta, variância extraída e $p$-valores encontrados, é possível validar o modelo de mensuração delineado. As variáveis latentes criadas, assim como as variáveis observadas que lhes deram origem, são apresentadas no Quadro 1.

Quadro 2. Variáveis latentes e observadas.

\begin{tabular}{|c|c|}
\hline Variáveis latentes & Variáveis observadas \\
\hline \multirow{5}{*}{ Flexibilização do trabalho } & FLEX1: Tenho possibilidade de realizar parte das minhas atividades de trabalho em casa. \\
\hline & $\begin{array}{l}\text { FLEX2: A organização onde trabalho adota mecanismos que permitem que os } \\
\text { funcionários realizem parte de suas atividades à distância. }\end{array}$ \\
\hline & $\begin{array}{l}\text { FLEX3: Minha rotina de trabalho permite que eu me desloque para a organização apenas } \\
\text { em dias específicos, pois posso realizar minhas tarefas fora dela. }\end{array}$ \\
\hline & $\begin{array}{l}\text { FLEX4: Tenho flexibilidade para alterar minha jornada de trabalho diária (horário de } \\
\text { entrada e saída). }\end{array}$ \\
\hline & FLEX5: Posso definir minha carga horária de trabalho diária, desde que eu cumpra a \\
\hline
\end{tabular}




\begin{tabular}{|c|c|}
\hline & carga horária de trabalho pré-estabelecida pela organização. \\
\hline & FLEX6: Tenho autonomia para definir meu horário de trabalho. \\
\hline & $\begin{array}{l}\text { FLEX7: No dia a dia tenho abertura para negociar alterações no meu horário de trabalho } \\
\text { com meus superiores. }\end{array}$ \\
\hline & $\begin{array}{l}\text { FLEX8: A organização permite a realização de jornada de trabalho diferenciada em } \\
\text { casos especiais, tais como: estudo, doença, maternidade etc. }\end{array}$ \\
\hline \multirow{3}{*}{ Satisfação do trabalhador } & SAT1: Meu trabalho é agradável. \\
\hline & SAT2: Gosto do que eu faço no trabalho. \\
\hline & SAT3: Sinto orgulho do meu trabalho. \\
\hline \multirow{5}{*}{ Foco em melhoria } & $\begin{array}{l}\text { CCO9: Faço sugestões construtivas para melhorar a forma como as coisas funcionam } \\
\text { dentro da organização. }\end{array}$ \\
\hline & CCO10: Procuro implantar soluções para resolução dos problemas organizacionais. \\
\hline & CCO8: Procuro instituir novos métodos de trabalho que são mais eficazes. \\
\hline & $\begin{array}{l}\text { CCO7: Procuro adotar procedimentos melhores para o departamento ou setor onde } \\
\text { trabalho. }\end{array}$ \\
\hline & CCO6: Faço sugestões inovadoras para melhorar o departamento ou setor onde trabalho. \\
\hline \multirow{4}{*}{ Conscienciosidade } & CCO13: Nunca saio mais cedo sem permissão. \\
\hline & CCO11: Nunca me atraso sem permissão prévia. \\
\hline & CCO12: Nunca deixo de seguir as instruções do meu supervisor. \\
\hline & CCO14: Sempre sigo as regras e procedimentos definidos pela empresa. \\
\hline \multirow{3}{*}{ Disposição para ajudar } & CCO3: Estou sempre pronto para ajudar meus colegas de trabalho. \\
\hline & CCO4: Ofereço-me voluntariamente para ajudar os outros na organização. \\
\hline & $\begin{array}{l}\text { CCO5: Ofereço-me voluntariamente para ajudar colegas com problemas relacionados } \\
\text { ao trabalho na organização. }\end{array}$ \\
\hline
\end{tabular}

Fonte: Elaboração própria.

Em seguida, examinaram-se as relações testadas no modelo estrutural, utilizando-se como parâmetros os pvalores obtidos e os coeficientes beta $(\beta)$ retornados pelo software AMOS.

Primeiramente, analisou-se a relação entre a flexibilização do trabalho e a satisfação do trabalhador, que obteve p-valor de 0,000 , indicando sua significância estatística a um nível de confiança de 95\%. Como o coeficiente beta foi de 0,323, verifica-se que se trata de uma relação diretamente proporcional, ou seja, quanto maior a flexibilização dos arranjos laborais, maior a satisfação experimentada pelo trabalhador. À vista disso, é possível ratificar $H_{l}$.

Na relação entre flexibilização do trabalho e foco em melhoria, obteve-se um p-valor de 0,436 . Sendo assim, não foi possível confirmar sua significância estatística e, por conseguinte, a influência dos arranjos laborais flexibilizados sobre a disposição do trabalhador de propor melhorias nos processos organizacionais e soluções para os problemas identificados.

Entre flexibilização do trabalho e conscienciosidade, observou-se uma relação estatisticamente significativa e inversamente proporcional, pois o p-valor obtido foi de 0,006 e o coeficiente beta foi de $-0,219$. Verifica-se, portanto, que os arranjos laborais flexíveis tendem a reduzir a disciplina do trabalhador para seguir as normas da organização e instruções de seu supervisor.

Da mesma forma, entre flexibilização do trabalho e disposição para ajudar, constatou-se que existe uma relação estatisticamente significativa e inversamente proporcional, à medida que se obteve p-valor de $0,032 \mathrm{e}$ coeficiente beta de $-0,213$. Nota-se, então, que os arranjos laborais flexíveis tendem a reduzir a solicitude dos indivíduos perante seus colegas de trabalho.

Analisando conjuntamente as duas últimas relações supracitadas, constata-se que a flexibilização dos arranjos 
laborais impacta negativamente no comportamento de cidadania organizacional dos trabalhadores, o que contraria a $\mathrm{H}_{3}$ inicialmente proposta.

Por sua vez, a relação entre satisfação do trabalhador e disposição para ajudar mostrou-se positiva e estatisticamente significativa, com p-valor igual a 0,000 e coeficiente beta de 0,578 . Logo, quanto maior a satisfação experimentada pelo indivíduo em seu trabalho, maior a solicitude demonstrada por ele perante seus pares na organização.

De modo semelhante, as relações entre satisfação do trabalhador e conscienciosidade e entre satisfação do trabalhador e foco em melhoria mostraram-se estatisticamente significativas e diretamente proporcionais, à medida que foram obtidos, respectivamente, $p$-valores de 0,001 e 0,000 e coeficientes beta de 0,262 e 0,621. Logo, verificase que os indivíduos satisfeitos com suas atividades laborais tendem a ser mais disciplinados quanto às normas organizacionais e instruções da chefia e mais dispostos a proporem melhorias nos processos organizacionais e soluções para os problemas identificados.

Analisando de forma concomitante as três últimas relações testadas, confirma-se que a satisfação do trabalhador afeta positivamente o comportamento de cidadania organizacional, o que ratifica $\mathrm{H}_{2}$.

Examinando os coeficientes de determinação associados ao modelo de equações estruturais proposto, verificou-se que a flexibilização do trabalho foi capaz de explicar 10,4\% da variação na satisfação dos trabalhadores, enquanto a flexibilização do trabalho e a satisfação do trabalhador explicaram a variação da disposição para ajudar em $30 \%$, do foco em melhoria em $41,4 \%$, e da conscienciosidade em $8 \%$, evidenciando-se o baixo poder explicativo do modelo estrutural relativo a essa última dimensão do comportamento de cidadania organizacional.

Finda a apresentação dos resultados alcançados por meio do tratamento estatístico dos dados coletados na presente pesquisa, importante se faz interpretá-los à luz do referencial teórico consultado.

A influência positiva exercida pela flexibilização dos arranjos laborais, que pode dar-se por meio de horários flexíveis, redução da jornada e/ou realização de parte do trabalho à distância (POSSENRIEDE; HASSINK; PLANTENGA, 2016), sobre a satisfação do trabalhador pode ser compreendida pela possibilidade que fornece ao sujeito de ajustar e melhor gerir suas responsabilidades profissionais e demandas pessoais (LU; CHANG, 2014), reduzindo seu nível de estresse e ampliando seu bem-estar e entusiasmo no cumprimento de suas funções (VIITALA; TANSKANEN; SÄNTTI, 2015).

Tal constatação encontra respaldo nos resultados obtidos por Humphrey, Nahrgang e Morgeson (2007), Cole, Oliver e Blaviesciunaite (2014), Possenriede, Hassink e Plantenga (2016), Possenriede e Plantenga (2014), Stavrou (2005) e Wheatley (2017), que indicaram que arranjos de trabalho mais flexíveis tornam os trabalhadores mais satisfeitos, à medida que lhes propiciam maior autonomia e melhor qualidade de vida.

Por sua vez, o impacto positivo que a satisfação experimentada pelo trabalhador exerce sobre o comportamento de cidadania organizacional explica-se pelo fato de que profissionais satisfeitos são mais produtivos e engajados, o que pode levar à apropriação de responsabilidades que extrapolam as obrigações descritas em seus cargos (BOATENG; OKOE; MENSAH, 2017). Além disso, os indivíduos satisfeitos com seu trabalho não só assumem atividades extras, como adotam condutas para ajudar outras pessoas, sem esperar retorno, internalizando o espírito coletivista (ARDA et al., 2017).

Tal resultado encontra respaldo nos resultados obtidos por Herminingsih e Kasuri (2018), Pio e Tampi (2018), Belwalkar, Vohra e Pandey (2018), Gyekye e Haybatollahi (2015), Jung e Yoon (2015) e Kaur, Kaur e Dhar (2015), que indicaram que a satisfação no trabalho é um relevante preditor do comportamento de cidadania organizacional.

Por fim, a influência negativa que a flexibilização dos arranjos laborais exerce sobre o comportamento de cidadania organizacional explica-se pela possibilidade de o indivíduo escolher como, onde e quando trabalhar, que pode levar à falta de visibilidade, interação e socialização entre os membros da organização (RAFALSKI; ANDRADE, 2015), reduzindo a solicitude e o suporte dispensados aos colegas de trabalho. Além disso, conforme se verifica em Rose (2016), o comportamento de cidadania organizacional é estimulado pela convivência e proximidade física entre os funcionários, que acabam prejudicadas pela flexibilização dos horários e locais de trabalho.

Tal constatação ainda pode ser explicada por um possível efeito da flexibilização laboral levantado por Martinez e Gómez (2013): a falta de oportunidades de capacitação recebidas pelo trabalhador que permanece menos tempo no espaço físico da organização. Nesse caso, à medida que o indivíduo acaba "esquecido" pelos 
gestores no que diz respeito a seu desenvolvimento profissional, seu comportamento de cidadania organizacional diminui.

\section{Considerações Finais}

Desde que o construto comportamento de cidadania organizacional foi introduzido na Ciência Administrativa em 1983, partindo-se daquilo que era entendido como cooperação espontânea por parte dos trabalhadores, distintos pesquisadores procuraram explicá-lo e relacioná-lo a diferentes fatores, visando aprimorar a compreensão a respeito do que leva os indivíduos a assumirem funções extras no âmbito das organizações. Com isso, numerosos aspectos passaram a ser testados como possíveis antecedentes do CCO; entretanto, muitos deles encontram-se, até o momento, pouco explorados.

Partindo dessa constatação, definiu-se como objetivo de pesquisa analisar como a flexibilização do trabalho influencia a satisfação do trabalhador e como ambas afetam o comportamento de cidadania organizacional. Cabe destacar que a relação entre esses três construtos ainda remete a uma lacuna conceitual na literatura e, portanto, carece de investigação sistemática.

Examinando-se os resultados obtidos após as etapas de coleta e análise de dados da presente pesquisa, constatou-se que o CCO é influenciado positivamente pela satisfação experimentada pelo trabalhador e negativamente pela flexibilização dos arranjos laborais.

Uma possível explicação para a primeira relação encontrada é que, quando os indivíduos estão satisfeitos em seu trabalho, tornam-se menos estressados, mais entusiasmados e, por conseguinte, mais produtivos; com isso, desenvolvem melhor as próprias funções e passam a estar mais propensos a assumirem tarefas que extrapolam suas obrigações formais, ajudando voluntariamente seus pares e ainda propondo melhorias nos processos de trabalho.

De modo complementar, para explicar a segunda relação encontrada, pode-se trabalhar com a hipótese de que, ao se flexibilizarem os arranjos laborais por meio de práticas como o home-office e a adoção de horários flexíveis, amplia-se a liberdade do sujeito, mas, ao mesmo tempo, cresce seu isolamento em relação aos demais profissionais; com isso, reduz-se a interação entre ele e seus pares, o que tende a torná-lo mais individualista e a afetar negativamente sua empatia e disposição de cooperar espontaneamente com os outros.

Além disso, outra hipótese que pode explicar essa relação negativa entre a flexibilização dos arranjos laborais e o CCO é que, como o profissional passa a trabalhar em horários flexíveis e muitas vezes fora do espaço físico da organização, acaba distanciando-se de seu supervisor e até mesmo de algumas rotinas laborais, o que o torna menos meticuloso no que se refere às instruções que recebe da chefia e às normas organizacionais.

Em contrapartida, constatou-se que a flexibilização do trabalho influencia positivamente a satisfação dos indivíduos, o que pode ser compreendido ao se considerar que os arranjos laborais flexíveis favorecem a conciliação entre as demandas pessoais, familiares e profissionais do sujeito, tornando-o mais satisfeito com cada uma das esferas de sua vida.

Analisando conjuntamente os resultados obtidos, pode-se apontar uma aparente inconsistência: ao mesmo tempo em que os arranjos laborais flexíveis reduzem o CCO, aumentam a satisfação do trabalhador, que, por sua vez, influencia positivamente o CCO. Todavia, tal contradição pode ser interpretada como um indicativo de que as organizações podem usufruir tanto das vantagens diretas da flexibilização do trabalho, como o maior contentamento dos profissionais, quanto das indiretas, como o CCO (neste caso, estimulado pela satisfação individual ligada às atividades laborais). Para isso, basta que implantem mecanismos para evitarem o isolamento social dos indivíduos beneficiados, promovendo a interação entre os funcionários e entre eles e a chefia.

A contribuição do presente estudo para a Ciência Administrativa reside no suporte que fornece para que se amplie a compreensão acerca dos antecedentes do comportamento de cidadania organizacional, favorecendo a construção de um arcabouço conceitual robusto a respeito do tema e, por conseguinte, o avanço deste campo de pesquisa. Além disso, contribui para que se clarifiquem os reais impactos da flexibilização dos arranjos laborais.

Ademais, como contribuição gerencial, destaca-se o potencial norteador dos resultados alcançados para que os gestores sejam capazes de promover relações laborais mais flexíveis, contando com funcionários mais satisfeitos e, simultaneamente, dispostos a atuarem como cidadãos organizacionais.

Para futuras pesquisas, sugere-se que sejam analisados alguns fatores, como supervisão abusiva e falta de oportunidades de crescimento profissional, que possam influenciar negativamente o CCO. Complementarmente, recomenda-se que seja investigado o impacto, sobre o comportamento humano no trabalho, de práticas gerenciais 
que tornam a assunção de responsabilidades extras uma obrigação dos funcionários, como as promoções baseadas nesse critério.

\section{Referências}

ARDA, O. A.; DELEN, D.; TATOGLU, E.; ZAIM, S. An analytic approach to assessing organizational citizenship behavior. Decision Support Systems, v. 103, p. 9-23, 2017.

BAEZA, M. A.; WANG, Y. J.; WANG, V. L. Analyzing organizational citizenship behavior in Mexico: A collectivist perspective. Global Business and Organizational Excellence, v. 37, n. 1, p. 16-27, 2017.

BAJZIKOVA, L.; SAJGAliKOVA, H.; WOJCAK, E.; POLAKOVA, M. Are Flexible Work Arrangements Attractive Enough for Knowledge-intensive Businesses? Procedia - Social and Behavioral Sciences, v. 99 , p. 771-783, 2013.

BATEMAN, T. S.; ORGAN, D. W. Job satisfaction and the good soldier: The relationship between affect and employee "citizenship". Academy of Management Journal, v. 26, n. 4, p. 587-595, 1983.

BELWALKAR, S.; VOHRA, V.; PANDEY, A. The relationship between workplace spirituality, job satisfaction and organizational citizenship behaviors - an empirical study. Social Responsibility Journal, v. 14, n. 2, p. 410-430, 2018.

BOATENG, H.; OKOE, A. F.; MENSAH, T. D. The relationship between human resource practices and knowledge sharing in service firms. Business Information Review, v. 34, n. 2, p. 74-80, 2017.

BRIEF, A. P. Attitudes in and around organizations. Thousand Oaks: Sage, 1998.

BYRNE, B.M. Structural Equation Modeling with Amos: basic concepts, applications, and programming. 3. ed. New York: Routledge, 2016.

CERIBELI, H. B.; FERREIRA, F. J. R. Uma análise da relação entre flexibilização do trabalho, comprometimento organizacional e intenção de permanência na organização. Journal Globalization, Competitiveness and Governability, v. 10, n. 3, p. 37-56, 2016.

COENEN, M.; KOK, R. A. W. Workplace flexibility and new product development performance: The role of telework and flexible work schedules. European Management Journal, v. 32, n. 4, p. 564-576, 2014.

COLE, R.; OLIVER, A.; BLAVIESCIUNAITE, A. The changing nature of workplace culture. Facilities, v. 32, n. 14, p. 786-800, 2014.

COOPER, D. R.; SCHINDLER, P. S. Business research methods. 12. ed. Nova York: McGraw-Hill Companies, 2014.

DE LA TORRE-RUIZ, J. M.; VIDAL-SALAZAR, M. D.; CORDÓN-POZO, E. Benefit flexibility and benefit satisfaction: does employee's personality matter? Personnel Review, v. 46, n. 1, p. 2-16, 2017.

FIGUEIREDO FILHO, D. B.; SILVA JÚNIOR, J. A. Visão além do alcance: uma introdução à análise fatorial. Opinião Pública, v. 16, n. 1, p. 160-185, 2010.

FORTE, S. H. A. C.; MATOS, L. A.; OLIVEIRA, O. V. Recursos, estratégia e vantagem competitiva no setor imobiliário: O caso da incorporadora penta. Amazônia, Oraganizações e Sustentabilidade, v. 4, n. 1, p. 15 $34,2015$.

FREITAS, H.; OLIVEIRA, M.; SACCOL, A. Z.; MOSCAROLA, J. O método de pesquisa survey. Revista de Administração da USP - RAUSP, v. 35, n. 3, p. 105-112, 2000.

GEVREK, D.; SPENCER, M.; HUDGINS, D.; CHAMBERS, V. I can’t get no satisfaction. Personnel Review, v. 46, n. 5, p. 1019-1043, 2017.

GUPTA, M.; SHAHEEN, M.; REDDY, P. K. Impact of psychological capital on organizational citizenship behavior. Journal of Management Development, v. 36, n. 7, p. 973-983, 2017.

GYEKYE, S. A., HAYBATOLLAHI, M. Organizational citizenship behavior: an empirical investigation of the 
impact of age and job satisfaction on Ghanaian industrial workers. International Journal of Organizational Analysis, v. 23, n. 2, p. 285-301, 2015.

HAIR JR, J. F.; BABIN, B.; MONEY, A. H.; SAMOUEL, P. Fundamentos de métodos de pesquisa em Administração. Porto Alegre: Bookman, 2005.

HAIR JR, J. F.; BLACK, W. C.; BABIN, B. J.; ANDERSON, R. E.; TATHAM, R. L. Análise multivariada de dados. 6. ed. Porto Alegre: Bookman, 2009.

HERMININGSIH, A.; KASURI, R. Personality, relation to job satisfaction and organizational citizenship behavior. European Research Studies Journal, v. 21, n. 3, p. 371-377, 2018.

HUMPHREY, S. E.; NAHRGANG, J. D.; MORGESON, F. P. Integrating motivational, social, and contextual work design features: A meta-analytic summary and theoretical extension of the work design literature. Journal of Applied Psychology, v. 92, n. 5, p. 1332-1356, 2007.

JENA, R. K.; GOSWAMI, R. Measuring the Determinants of Organizational Citizenship Behaviour. Global Business Review, v. 15, n. 2, p. 381-396, 2014.

JUNG, H. S.; YOON, H. H. Error management culture and turnover intent among food and beverage employees in deluxe hotels: the mediating effect of job satisfaction. Service Business, v. 11, n. 4, p. 785-802, 2017.

JUNG, H. S.; YOON, H. H. The impact of employees' positive psychological capital on job satisfaction and organizational citizenship behaviors in the hotel. International Journal of Contemporary Hospitality Management, v. 27, n. 6, p. 1135-1156, 2015.

KAUR, M.; KAUR, S. DHAR, N. A study of job satisfaction as a predictor of organizational citizenship behavior. Prabandhan: Indian Journal of Management, v. 8, n. 1, p. 34-45, 2015.

LEE, J. H. J.; OK, C. M.; HWANG, J. An emotional labor perspective on the relationship between customer orientation and job satisfaction. International Journal of Hospitality Management, v. 54, p. 139-150, 2016.

LIM, B. T. H.; LOOSEMORE, M. The effect of inter-organizational justice perceptions on organizational citizenship behaviors in construction projects. International Journal of Project Management, v. 35, n. 2, p. 95-106, 2017.

LU, L.; CHANG, Y.-Y. An integrative model of work/family interface for Chinese employees. Career Development International, v. 19, n. 2, p. 162-182, 2014.

LÜTKEMEYER FILHO, M. G.; ROEHEVACCARO, G. L.; DE FREITAS, E. C. Identificação dos fatores de satisfação de clientes em serviços: Um estudo em concessionárias do agronegócio. Revista Brasileira de Gestao de Negocios, v. 17, n. 58, p. 1408-1425, 2015.

MARINOVA, S. V; MOON, H.; VAN DYNE, L. Are all good soldier behaviors the same? Supporting multidimensionality of organizational citizenship behaviors based on rewards and roles. Human Relations, v. 63, n. 10, p. 1463-1485, 2010.

MARTIN, B.H.; MACDONELL, R. Is telework effective for organization? A meta-analysis of empirical research on perspections os telework and organizational outcomes. Management Research Review, v. 35, n. 7, p. 602-616, 2012.

MARTINEZ, P.; GÓMEZ, C. B. Trading telecommuting flexibility for fewer training opportunities? Management Research, v. 11, n. 3, p. 235-259, 2013.

MAYFIELD, J.; MAYFIELD, M. Step by step to better performance. Human Resource Management International Digest, v. 22, n. 4, p. 36-39, 2014.

MÁZARO, A. L.; LEAL, G. S.; ROSALEM, V.; SADOYAMA, A. S. P. Percepção da qualidade de serviços educacionais em instituição de ensino superior privada em Catalão, GO. Enciclopédia Biosfera, v. 10, n. 19, 
p. 379, 2014.

MCDONALD, P.; BRADLEY, L.; BROWN, K. "Full-time is a given here": Part-time versus full-time job quality. British Journal of Management, v. 20, n. 2, p. 143-157, 2009.

MIAO, C.; HUMPHREY, R. H.; QIAN, S. A meta-analysis of emotional intelligence and work attitudes. Journal of Occupational and Organizational Psychology, v. 90, n. 2, p. 177-202, 2017.

MICHEL, J. W. Antecedents of Organizational Citizenship Behaviors: Examining the Incremental Validity of SelfInterest and Prosocial Motives. Journal of Leadership \& Organizational Studies, v. 24, n. 3, p. 385-400, 2017.

MOCELIN, D. G. A Redução da Jornada de Trabalho e a qualidade dos Empregos: entre o Discurso, a Teoria e a Realidade. p. 101-119, 2011.

MONTERO, R.; VASQUEZ, D. Job satisfaction and reference wages: evidence for a developing country. Journal os Happiness Studies, v. 16, n. 6, p. 1493-1507, 2015.

MULDOON, J.; KEOUGH, S. M.; LIGUORI, E. W. The role of job dedication in organizational citizenship behavior performance. Management Research Review, v. 40, n. 10, p. 1042-1057, 2017.

NGUYEN, B.; CHANG, K.; ROWLEY, C.; JAPUTRA, A. Organizational citizenship behavior, identification, psychological contract and leadership frames. Asia-Pacific Journal of Business Administration, v. 8, n. 3, p. 260-280, 2016.

NOBACK, I.; BROERSMA, L.; VAN DIJK, J. Climbing the Ladder: Gender-Specific Career Advancement in Financial Services and the Influence of Flexible Work-Time Arrangements. British Journal of Industrial Relations, v. 54, n. 1, p. 114-135, 2016.

OLANIYAN, O. S.; HYSTAD, S. W. Employees' psychological capital, job satisfaction, insecurity, and intentions to quit: The direct and indirect effects of authentic leadership. Revista de Psicología del Trabajo y de las Organizaciones, v. 32, n. 3, p. 163-171, 2016.

PAUL, H.; BAMEL, U. K.; GARG, P. Employee resilience and OCB: Mediating effects of organizational commitment. Journal for Decision Makers, v. 41, n. 4, p. 308-324, 2016.

PIO, R. J.; TAMPI, J. R. E. The influence of spiritual leadership on quality of work life, job satisfaction and organizational citizenship behavior. International Journal of Law and Management, v.60, n. 2, p. 757$767,2018$.

PODSAKOFF, P. M.; MACKENZIE, S. B.; PAINE, J. B.; BACHRACH, D. G. Organizational Citizenship Behaviors: A critical review of the theorical and empirical literature and suggestions for future research. Journal os Management, v. 26, n. 3, p. 513-563, 2000.

POSSENRIEDE, D.; HASSINK, W. H. J.; PLANTENGA, J. Does temporal and locational flexibility of work increase the supply of working hours? Evidence from the Netherlands. IZA Journal of Labor Policy, v. 5, n. 1, p. 16, 2016.

POSSENRIEDE, D.; PLANTENGA, J. Temporal and Locational Flexibility of Work, Working-Time Fit, and Job Satisfaction. IZA Discussion Paper, n. 8436, 2014.

RAFALSKI, J. C.; ANDRADE, A. L. Home-office: aspectos exploratórios do trabalho a partir de casa. Temas em Psicologia, v. 23, n. 2, p. 431-441, 2015.

READER, T. W. et al. Organizational support for the workforce and employee safety citizenship behaviors: A social exchange relationship. Human Relations, v. 70, n. 3, p. 362-385, 2017.

ROBERTSON, J. L.; BARLING, J. Toward a new measure of organizational environmental citizenship behavior. Journal of Business Research, v. 75, p. 57-66, 2017. 
ROSE, K. Examining Organizational Citizenship Behavior in the Context of Human Resource Development. Human Resource Development Review, v. 15, n. 3, p. 295-316, 2016.

SANTOS, A. B.; ANTONELLI, S. C. Aplicação da abordagem estatística no contexto da gestão da qualidade: um survey com indústrias de alimentos de São Paulo. Gestão da Produção, v. 18, n. 3, p. 509-524, 2011.

SCHUMACKER, R. E; LOMAX, R. G. A beginner's guide to structural equation modeling. 3. ed. New York: Routledge, 2010.

SMITH, C. A.; ORGAN, D. W.; NEAR, J. P. Organizational citizenship behavior: Its nature and antecedents. Journal of Applied Psychology, v. 68, n. 4, p. 653-663, 1983.

SPECTOR, P. E. Psicologia nas organizações. São Paulo: Saraiva, 2006.

STACCIARINI, T. S. G.; PACE, A. E. Análise fatorial confirmatória da escala: appraisal of self care agency scale - revised. Revista Latino-Americana de Enfermagem, v. 25, 2017.

STAVROU, E. T. Flexible work bundles and organizational competitiveness: A cross-national study of the European work context. Journal of Organizational Behavior, v. 26, n. 8, p. 923-947, 2005.

TUNA, M.; GHAZZAWI, I.; YESILTAS, M.; TUNA, A.; ARSLAN, S. The effects of the perceived external prestige of the organization on employee deviant workplace behavior. The mediating role of job satisfaction. International Journal of Contemporary Hospitality Management, v. 28, n. 2, p. 366-396, 2016.

VIANA, A. B. N. Pesquisa quantitativa aplicada ao varejo. In: MERLO, E. M. (Org.). Administração de varejo com foco em casos brasileiros. Rio de Janeiro: LTC, 2011.

VIITALA, R.; TANSKANEN, J.; SÄNTTI, R. The connection between organizational climate and well-being at work. International Journal of Organizational Analysis, v. 23, n. 4, p. 606-620, 2015.

WALTER, O. M. F. C. Análise de ferramentas gratuitas para condução de survey online. Produto \& Produção, v. 14, n. 2, p. 44-58, 2013.

WANG, L.; HINRICHS, K. T.; PRIETO, L.; HOWELL, J.P. Five dimensions of organizational citizenship behavior: Comparing antecedents and levels of engagement in China and the US. Asia Pacific Journal of Management, v. 30, n. 1, p. 115-147, 2013.

WEIKAMP, J. G.; GÖRITZ, A. S. Organizational citizenship behaviour and job satisfaction: The impact of occupational future time perspective. Human Relations, v. 69, n. 11, p. 2091-2115, 2016.

WHEATLEY, D. Work-life balance, travel-to-work, and the dual career household. Personnel Review, v. 41, n. 6 , p. 813-831, 2012.

WHEATLEY, D. Employee satisfaction and use of flexible working arrangements. Work, employment and society, v. 31, n. 4, p. 567-585, 2017.

WHITMAN, D. S.; VAN ROOY, D. L.; VISWESVARAN, C. Satisfaction, citizenship behaviors, and performance in work units: A meta-analysis of collective construct relations. Personnel Psychology, v. 63, n. 1, p. 41-81, 2010.

WILLIAMS, L. J.; ANDERSON, S. E. Job satisfaction and organizational commitment as predictors of organizational citizenship behaviors. Journal os Management, v. 17, n. 3, p. 601-617, 1991.

WONG, I. K. A.; WAN, Y. K. P.; GAO, J. H. How to attract and retain Generation Y employees? An exploration of career choice and the meaning of work. Tourism Management Perspectives, v. 23, p. 140-150, 2017.

YADAV, M.; RANGNEKAR, S.; BAMEL, U. Workplace Flexibility Dimensions as Enablers of Organizational Citizenship Behavior. Global Journal of Flexible Systems Management, v. 17, n. 1, p. 41-56, 2016.

ZHANG, S. Impact of Job Involvement on Organizational Citizenship Behaviors in China. Journal of Business Ethics, v. 120, n. 2, p. 165-174, 2014. 
ZHANG, Y.; GUO, Y.; NEWMAN, A. Identity judgements, work engagement and organizational citizenship behavior: The mediating effects based on group engagement model. Tourism Management, v. 61, p. 190197, 2017. 